\title{
Refining Surface Curvature with Relaxation Labeling
}

\author{
Richard C. Wilson and Edwin R. Hancock \\ Department of Computer Science \\ University of York, York, Y01 5DD, UK.
}

\begin{abstract}
Our main contributions in this paper are twofold. In the first instance, we demonstrate how $H-K$ surface labelling can be realised using dictionary-based probabilistic relaxation. To facilitate this implementation we have developed a dictionary of feasible surface-label configurations. These configurations observe certain constraints on the contiguity of elliptic and hyperbolic regions, and, on the continuity and thinness of parabolic lines. The second contribution is to develop a statistical model which allows scheme to be initialised using the probabilities of the different $H-K$ labels to be estimated from surface normal information.
\end{abstract}

\section{Introduction}

Curvature labels provide a natural way of describing the intrinsic differential structure of surfaces. Mean and Gaussian curvature labels derived from the eigen-structure of the Hessian matrix allow surfaces to be segmented into meaningful structures such as ridges or valleys, saddle points or lines, and, domes or cups. These structures can be further organised into simply connected elliptical or hyperbolic regions which are separated from one-another by parabolic lines. Unfortunately, because the Hessian matrix is based on second-derivatives the reliable estimation of surface curvature has proved to be a task of notorious difficulty in the analysis and range or volumetric imagery $[8,9]$. Some of the limitations of the alternative strategies for curvature estimation were unearthed in the comparative study of Flynn and Jain [3].

It is for these reasons that strategies aimed at circumventing the direct estimation of second-derivatives have been developed. One of the most popular approaches is to approximate the surface by a low-order piecewise continuous surface [5,2]. For instance, Besl and Jain adopt a hierarchical fitting technique [1]. Firstly, a local tangent plane is extracted by identifying the principal component axes for the surface point distribution over a support neighbourhood, Next, the plane-fit is refined using a cubic patch. Hilton, Illingworth and Windeat [6] have addressed the issue of analysis of variance to improve the statistical fidelity of the fitting process.

Despite these efforts at improving the reliability of curvature estimation the problem of how to refine inconsistent curvature estimates has received less attention. In essence, surface-fitting does not guarantee that the extracted estimates 
of the Hessian are consistent when viewed from the requirements that elliptic and hyperbolic regions should be simply connected, or, that parabolic lines should be thin and continuous. Interrogation of the literature reveals that it is only Sander and Zucker [10] who have made any serious attempt at exploiting the idea of curvature consistency to improve the recovery of a consistent surface description. Their idea has been to iteratively update Darboux frames by imposing the constraint that the principal curvature directions should vary smoothly across the surface. The initial estimates of the Hessian required in this analysis are derived from the least squares fitting of bi-quadric patches. However, there is no attempt to reconcile the quality of the recovered surface description with the underlying statistical uncertainties in the raw surface data. Neither is there any attempt to exploit the structured nature of the $H-K$ surface-labels in improving curvature consistency.

Our overall aim in this paper is to present a statistical framework for surface curvatur labelling. We commence by showing how the Hessian matrix can be directly estimated using statistics derived from surface normals. Our motivation in embarking on this statistical analysis is to realise the process of consistent curvature-label refinement using probabilistic relaxation labelling. The framework adopted in this study is the dictionary-based relaxation scheme of Hancock and Kittler [4]. The critical ingredient is a dictionary which represents the valid configurations of HK curvature labels that can be consistently assigned to neighbouring sites on the surface. In this way we tap the rich source of constraints provided by the highly structured nature of surface curvature labels.

\section{Representing Differential Surface Structure}

In this paper we are interested in estimating the local differential structure of surfaces using computed estimates of the surface normal directions. This is to be contrasted with the fitting of a local surface patch and estimating curvature from the computed parameters of the patch We commence by providing some of the formal ingredients of our surface representation. The local surface orientation is determined by the direction of the surface normal $\mathbf{n}=\left(n_{x}, n_{y}, 1\right)^{T}$. When the surface is represented by a twice differentiable function $z=f(x, y)$, then the components of the normal are related to the surface gradient, i.e. $\mathbf{n}=\left(\frac{\partial f}{\partial x}, \frac{\partial f}{\partial y}, 1\right)^{T}$. In this continuous case, the differential structure of the surface is captured by the Hessian matrix

$$
\mathcal{H}=\left(\begin{array}{cc}
\frac{\partial^{2} f}{\partial x^{2}} & \frac{\partial^{2} f}{\partial x \partial y} \\
\frac{\partial^{2} f}{\partial x \partial y} & \frac{\partial^{2} f}{\partial y^{2}}
\end{array}\right)
$$

The eigen-structure of the Hessian matrix can be used to gauge the curvature of the surface. The two eigen-values of $\mathcal{H}$ are the maximum and minimum curvatures. The orthogonal eigen-vectors of $\mathcal{H}$ are known as the principal curvature directions. The mean-curvature of the surface is found by averaging the maximum and minimum curvatures. The Gaussian curvature is equal to the product of the two eigenvalues. 
In the case when surface normal information is being used to characterise the surface, then the Hessian matrix takes on the following form

$$
\mathcal{H}=\left(\begin{array}{ll}
\alpha & \beta \\
\beta & \gamma
\end{array}\right)
$$

The diagonal elements of the Hessian are related to the rate-of change of the surface normal components, i.e., $\alpha=\frac{\partial n_{x}}{\partial x}$ and $\gamma=\frac{\partial n_{\Phi}}{\partial x}$. Treatment of the offdiagonal elements is more subtle. However, under the assumption that the surface is locally developable then we can write $\beta=\frac{\partial n_{x}}{\partial y}=\frac{\partial n_{y}}{\partial x}$. In the next Section we will describe how the elements of the Hessian, i.e. $\alpha, \beta$ and $\gamma$, can be estimated from raw surface normal data using the method of least-squares.

With estimates of the elements of the Hessian to-hand, we can compute the mean $(\mathrm{K})$ and Gaussian $(\mathrm{H})$ curvatures of the surface. According to the definitions given above $K=\frac{1}{2}(\alpha+\gamma)$ and $H=\alpha \gamma-\beta^{2}$. The signs and zeros of these two quantities can be used to label the surface according to curvature class. The different classes are defined in Table 1. It is important to stress that there are adjacency constraints applying to the curvature labels. In particular, the the cup (C) and dome (D) surface types may not appear adjacent to each other on a surface. Moreover, elliptic regions on the surface (those for which $H$ is positive) must be separated from hyperbolic regions (those for which $H$ is negative) by a parabolic line (where $H=0$ ). In other words, domes and cups are enclosed by ridge or valley-lines. Moreover, domes or cups can not be adjacent to saddlestructures. In Section 5 we will exploit these constraints to construct a dictionary for the $H-K$ curvature labels.

\begin{tabular}{||c|c|c|c|c||}
\hline Class & Symbol & $\mathrm{K}$ & $\mathrm{H}$ & Region-type \\
\hline Dome & $\mathrm{D}$ & - & + & Elliptic \\
Ridge & $\mathrm{R}$ & - & 0 & Parabolic \\
Saddle ridge & $\mathrm{SR}$ & - & - & Hyperbolic \\
Plane & $\mathrm{P}$ & 0 & 0 & Hyperbolic \\
Saddle-point & $\mathrm{S}$ & 0 & - & Hyperbolic \\
Cup & $\mathrm{C}$ & + & + & Elliptic \\
Valley & $\mathrm{V}$ & + & 0 & Parabolic \\
Saddle-valley & $\mathrm{SV}$ & + & - & Hyperbolic \\
\hline
\end{tabular}

Table 1. Curvature classes

\section{Computing the Hessian using Sampled Normals}

In this section we describe how to make a statistical estimate of the Hessian matrix from a sample of surface normals. Specifically, we use the method of least squares to estimate the elements of $\mathcal{H}$ and to compute the errors associated with these estimates.

We commence by assuming that we have a set of surface normal measurements associated with a tentative surface. Moreover, we assume that the variance in the surface normal components is known. For instance, in the case of volumetric intensity images with the surface normals estimated using directional 
edge-detection operators, then Sharp and Hancock [11] have shown how the variance-covariance matrix for the surface normals is determined by the intensity noise-variance together with the autocorrelations of the filter kernels. Let $\mathbf{n}_{0}$ represent the surface normal at the position $\left(x_{0}, y_{0}, z_{0}\right)$. and let $\mathbf{n}_{m}$ be a neighbouring surface normal with position $\left(x_{m}, y_{m}, z_{m}\right)$. If the normals are close to each other, then we can approximate the change in the components of the surface normal using a first-order Taylor expansion. Accordingly, $\Delta n_{x}=\frac{\partial n_{x}}{\partial x} \Delta x+\frac{\partial n_{x}}{\partial y} \Delta y$ and $\Delta n_{y}=\frac{\partial n_{y}}{\partial x} \Delta x+\frac{\partial n_{y}}{\partial y} \Delta y$, where the measured change in the components of the surface normal is given by $\mathbf{n}_{m}-\mathbf{n}_{0}=\left(\Delta^{m} n_{x}, \Delta^{m} n_{y}, 0\right)^{T}$. The displacements in point co-ordinates are $\Delta^{m} x=x_{m}-x_{0}$ and $\Delta^{m} y=y_{m}-y_{0}$. With these relationships to hand we can rewrite the Taylor expansion in terms of elements of the Hessian matrix, i.e. $\Delta^{m} n_{x}=\alpha \Delta^{m} x+\beta \Delta^{m} y$ and $\Delta^{m} n_{y}=\beta \Delta^{m} x+\gamma \Delta^{m} y$. These equations govern the parallel transport of the vector across the curved geometry of the surface. So, to first-order, the change in the normal is linear in the elements of the Hessian matrix. Unfortunately, for the single neighbouring normal these equations are under-constrained and we can not recover the Hessian. However, if we have a sample of $N$ neighboring surface normals, then there are $2 N$ linear homogenous equations in the elements of $\mathcal{H}$ and the problem of recovering differential structure is no-longer under-constrained. We make the homogeneous nature of the equations more explicit by writing

$$
\begin{aligned}
& \Delta n_{x}^{(m)}=\Delta x^{(m)} \cdot \alpha+\Delta y^{(m)} \cdot \beta+\quad 0 \cdot \gamma \\
& \Delta n_{y}^{(m)}=0 \cdot \alpha+\Delta x^{(m)} \cdot \beta+\Delta y^{(m)} \cdot \gamma
\end{aligned}
$$

In order to simplify notation, we can write the full system of $2 \mathrm{~N}$ equations in matrix form as $\mathbf{N}=\mathbf{X P}$, where $\mathbf{N}$ is an aggregated column-vector of normal components, i.e. $\mathrm{N}=\left(\Delta n_{x}^{(1)}, \Delta n_{y}^{(1)}, \Delta n_{x}^{(2)}\right)^{T}$. The design matrix $\mathrm{X}$ represents the co-ordinate displacements

$$
\mathbf{X}=\left(\begin{array}{ccc}
\Delta x^{(1)} & \Delta y^{(1)} & 0 \\
0 & \Delta x^{(1)} & \Delta y^{(1)} \\
\Delta x^{(2)} & \Delta y^{(2)} & 0 \\
\vdots &
\end{array}\right)
$$

and the parameter vector $\mathbf{P}=(\alpha, \beta, \gamma)^{T}$. When the system of equations is overspecified in this way, then we can extract the set of parameters that minimises the vector of error-residuals $\mathbf{N}-\mathbf{X P}$. We pose this parameter recovery process as a least-squares estimation problem. In other words we seek the set of estimated parameters $\hat{\mathbf{P}}=(\hat{\alpha}, \hat{\beta}, \hat{\gamma})^{\mathbf{T}}$ which satisfy the condition $\hat{\mathbf{P}}=\arg \min _{\mathbf{P}}(\mathbf{N}-$ $\mathbf{X P})^{\mathbf{T}}(\mathbf{N}-\mathbf{X P})$. The solution-vector is given by $\hat{\mathbf{P}}=\left(\mathbf{X}^{\mathbf{T}} \mathbf{X}\right)^{-1} \mathbf{X}^{\mathbf{T}} \mathbf{N}$. The leastsquares estimates of the parameters can then be used to compute the set of surface labels. 


\section{Labelling the surface}

In order exploit the highly structured nature of the surface labelling constraints, we have chosen to employ the technique of dictionary brased probabilistic relaxation. Our reasons for this are twofold. In the first instance, the method draws on labelling constraints using an exhaustive list or compilation of valid neighbourhood label configurations. This list is referred to as a dictionary. The second reason is that the framework is Bayesian and combines evidence for label assignments. Rather than commencing from hard and potentially erroneous label assignments, the initial characterisation is in terms of a posteriori label probabilities. These initial probabilities are computed from distribution functions which characterise uncertainties in the unary attributes from which label decisions are to be derived. In other words, dictionary-based relaxation allows us to exploit both consistent surface label structure and the covariance structure of the $\mathrm{H}-\mathrm{K}$ curvatures.

According to the original formulation of Hancock and Kittler [4], the local labelling is described by a set of probabilities. Specifically, $P^{(n)}\left(S_{i}=\omega\right)$ is the weight of evidence assigned to the label assignment $\omega$ at site $S_{i}$ for the iterative epoch $n$ of the algorithm. These weights are initialised with the probability that label $\omega$ takes on one of the eight possibilities from Table 1. Initially, these probabilities are calculated using the computed values of $H$ and $K$, together with their known covariance structure.

These label-probabilities are iteratively updated using the non-linear relaxation rule

$$
P^{(n+1)}\left(S_{i}=\omega\right)=\frac{P^{(n)}\left(S_{i}=\omega\right) Q^{(n)}\left(S_{i}=\omega\right)}{\sum_{\omega^{\prime} \in \Omega} P^{(n)}\left(S_{i}=\omega^{\prime}\right) Q^{(n)}\left(S_{i}=\omega^{\prime}\right)}
$$

The critical ingredient in the update formula is the support function $Q^{(n)}\left(S_{i}=\right.$ $\omega)$ which combines evidence from the context-conveying neighbourhood $K_{i}$ of the surface-site $S_{i}$ for the label assignment $\omega \in \Omega$. Here $\Omega=\{D, R, S R, S, S V, V, C\}$ denotes the complete set of curvature labels. According to Hancock and Kittler [4], the support function takes on the following product-form

$$
Q^{(n)}\left(S_{i}=\omega\right)=\sum_{\Lambda \in \Theta(\omega)}\left\{\prod_{k \in K_{i}} \frac{P^{(n)}\left(S_{k}=\lambda_{k}\right)}{P\left(S_{k}=\lambda_{k}\right)}\right\} P\left(S_{l}=\lambda_{l} \forall l \in K_{i}\right)
$$

In the above formula the dictionary $\Theta(\omega)$ contains a set of legitimate labellings over this neighbourhood $K_{i}$. The dictionary item $A=\left\{\lambda_{k} ; k \in K_{i}\right\}$ is a configuration of valid curvature labels on the neighbourhood $K_{i}$.

\section{Dictionary}

As mentioned above, there are strong adjacency constraints on the valid label configurations appearing on the neighbourhoods of some classes of surface. One 
of the goals of the work reported in this paper is to describe a methodology for enumerating and encoding these constraints in a dictionary for $H-K$ labels. Of course, the construction of a dictionary depends critically on the choice of neighbourhood topology. In the experimental section later we will be demonstrating the utility of the method on triangular surface meshes. In this case the natural neighbourhood consists of a triangle and it's three directly adjoining elements. We will therefore confine our attention exclusively to this arrangement of objects in constructing the dictionary.

We commence by considering the dome-class (D) for which $H>0$ and $K>0$. This is an example of an elliptic region. It can therefore be connected to other dome labels. It can not co-occur with any class other than the ridge for which $H=0$ and $K>0$. The configurations satisfying these two constraints are shown in Figure 1. It should be noted that all sections of the dictionary are rotation invariant. The cup-class $(\mathrm{C})$ is symmetric with the dome under reversal of the sign of the mean curvature $(\mathrm{K})$. Under this transformation, the ridge-label is replaced with the valley label. The dictionary for the cup class is therefore constructed by performing the mappings $D \rightarrow C$ and $R \rightarrow V$ in Figure 1 .
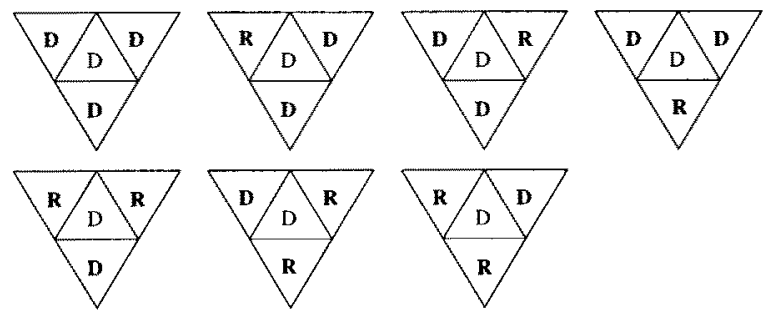

Fig. 1. Elements of the dictionary for the D-label

The two hyperbolic region labels have a more complicated neighbourhood structure. The saddle-valley and the saddle-ridge labels can again form contiguous patches. However, they can be bounded by both the saddle-point and the parabolic line of appropriate mean-curvature. For instance the saddle-ridge can be adjacent to the ridge and the saddle-point. The ridge and valley labels fall into the category of parabolic lines. In other words, they must form the boundaries between hyperbolic regions and elliptical regions. Specifically, they are effectively zero crossings of Gaussian curvature. In consequence the ridge label intercedes between elliptical domes and hyperbolic saddle-ridges. The dictionaries for these hyperbolic and parabolic label classes can be compiled in an analogous manner to Figure 1, but are omitted here due to space limitations.

\section{Experimental evaluation}

In this section we offer some experimental validation of our surface-labelling algorithm. In order to evaluate the method under controlled conditions, the ex- 
periments are conducted using synthetic surfaces. There surfaces are subjected to controlled levels additive Gaussian noise. The surfaces simulate range images. The additive Gaussian noise models sensing errors in the surface height distribution. The raw height data is triangulated using sample points from the surface. These triangles then provide surface normal information.

Figure 2a shows the initial labelling of a damped-cosine surface. The surface labels are shaded according to a convention in which cups, domes, saddle valleys and saddle ridges apear as progressively light regions. Notice that the initial labelling contains no parabolic line-structure. In other words, the abutting boundaries of the different regions are intrinsically inconsistent when viewed from the perspective of the H-K label-set. Figure $2 \mathrm{~b}$ shows the labelling of the surface after 6 iterations of the dictionary-based relaxation scheme. The green triangles appearing in the updated labelling are parabolic lines (i.e. either ridges or valleys). Although there are sampling artifacts due to the triangular elements used in our labelling scheme, the parabolic lines are thin and continuous. More significantly, they delineate elliptic and hyperbolic regions. Finally, Figure 3 shows the labelling of two noisy damped-cosine surfaces $(\sigma=0.5$ and $\sigma=1.0$ respectively).
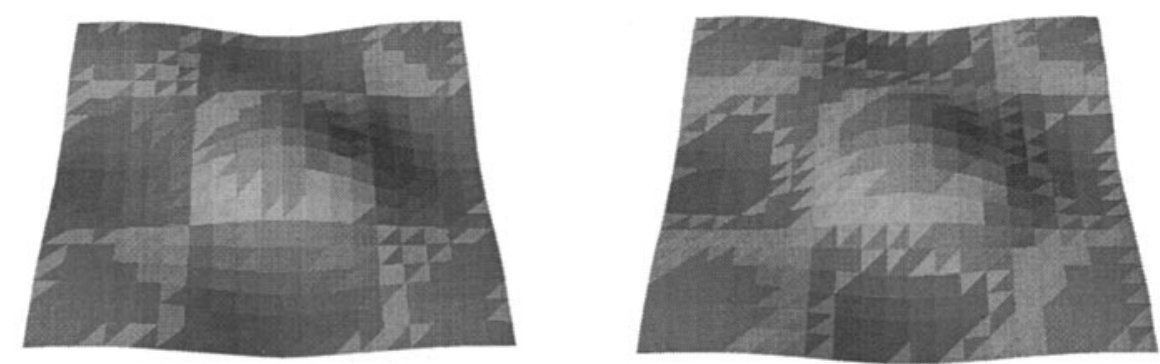

Fig. 2. Initial (left) and final (right) labellings of damped-cosine surface

\section{Conclusions}

Our main contributions in this paper are twofold. In the first instance, we have demonstrated how $H-K$ surface labelling can be realised using dictionary-based probabilistic relaxation. To facilitate this implementation we have developed a dictionary of feasible surface-label configurations. These configurations observe certain constraints on the contiguity of elliptic and hyperbolic regions, and, on the continuity and thinness of parabolic lines. The second contribution has been to develop a statistical model which allows curvature lables to be estimated using surface normal statistics. 

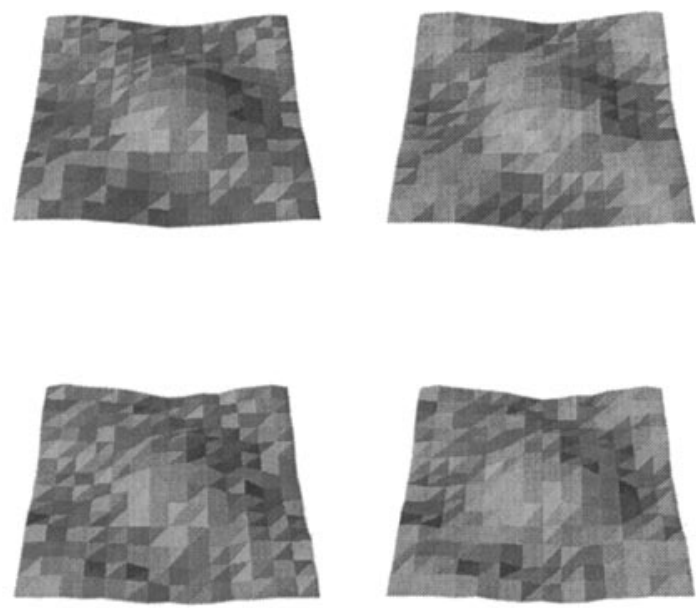

Fig. 3. Initial and final labelling two noisy damped-cosine surfaces

\section{References}

1. Besl P.J. and Jain R.C., "Segmentation through variable order surface fitting", IEEE PAMI, PAMI 10, pp 167-192, 1988.

2. Bolle R. M. and Cooper D.B, "Bayesian recognition of of local 3D shape by approximating image intensity functions with quadric polynomials", IEEE $P A M I, 6$, pp. 418-429, 1984.

3. Flynn P.J., and Jain A.K., "On reliable curvature estimation", IEEE Computer Vision and Pattern Recognition Conference, pp. 110-116, 1989.

4. Hancock E.R. and Kittler J, "Edge labelling using dictionary based relaxation", IEEE PAMI, PAMI 12, pp.165-181, 1990.

5. Haralick R.M. and Watson L., "A facet model for image data", Computer Graphics and Image Processing, 15, pp. 115-129, 1981.

6. Hilton A., Illingworth J. and Windeatt T., "Statistics of surface curvature estimates", Pattern Recognition, 28, pp. 1201-1221, 1995.

7. Koenderinck J.J. and Van Doorn A., "Surface shape and curvature scales", Image and Vision Computing, 10, pp. 557-564, 1992

8. Monga O., Deriche R., and Malandain G., "Recursive Filtering and edge closing; two primary tools for $3 \mathrm{D}$ edge detection", Image and Vision Computing, 9, pp. , 1991.

9. Monga O. and Benayoun S., "Using differential geometry in R4 to extract typical features in 3D density images", 11th International Conference on Pattern Recognition, Volume 1, pp 379-382, 1992.

10. Sander P.T. and Zucker S.W., "Inferring surface structure and differential structure from 3D images", IEEE PAMI, PAMI 12, pp 833-854, 1990.

11. Sharp N.G. and E. R. Hancock, "Feature Tracking by Multi-frame Relaxation", Image and Vision Computing, 13, pp. 637-644, 1995.

12. Wilson R.C. and Hancock E.R., "A minimum variance surface mesh", submitted, 1997. 